\title{
Improving Parameters Selection of a Seeded Region Growing Method for Multiband Image Segmentation
}

\author{
J. Sánchez, E. Martínez and A. Arquero
}

\begin{abstract}
In the last decade, Object Based Image Analysis (OBIA) has been accepted as an effective method for processing high spatial resolution multiband images. This image analysis method is an approach that starts with the segmentation of the image. Image segmentation in general is a procedure to partition an image into homogenous groups (segments). In practice, visual interpretation is often used to assess the quality of segmentation and the analysis relies on the experience of an analyst. In an effort to address the issue, in this study, we evaluate several seed selection strategies for an automatic image segmentation methodology based on a seeded region growing-merging approach. In order to evaluate the segmentation quality, segments were subjected to spatial autocorrelation analysis using Moran's $I$ index and intra-segment variance analysis. We apply the algorithm to image segmentation using an aerial multiband image.
\end{abstract}

\section{INTRODUCCION}

$\mathrm{E}^{\mathrm{L}}$ ANÁLISIS de imágenes orientado a objetos se ha convertido desde hace algún tiempo, en una tendencia ampliamente utilizada en las imágenes registradas por sensores trasportados en plataformas aeroespaciales [1]. Esto es debido a que el análisis tradicional orientado a píxeles no da buenos resultados en imágenes de alta resolución espacial [2], propiedad que presentan los sensores más avanzados tecnológicamente. Estos sensores constituyen la principal fuente de adquisición de información en el ámbito de la Teledetección, siendo esta tecnologia uno de los principales orígenes de los datos para los Sistemas de Información Geográfica (SIG) [3]

Una vez que se han adquirido las imágenes y se han preprocesado, la etapa fundamental del análisis de imágenes orientado a objetos (Object Based Image Analysis, OBIA), en Teledetección. es la segmentación de dichas imágenes [1]. La segmentación es un proceso tradicionalmente utilizado en procesado de imagen y consiste en la partición de la imagen en objetos o regiones separadas [4]. También y con frecuencia se aplican algoritmos optimizados basados en segmentación sobre otros tipos de imágenes como son las biomédicas [5-9] obteniendo buenos resultados.

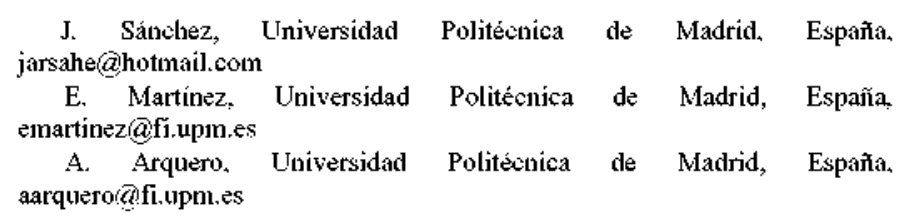

Tradicionalmente, los métodos de segmentación pueden ser agrupados en: los basados en pixel, los basados en bordes, los basados en regiones y los mixtos. En el primer caso, la segmentación se basa en valores umbrales que son seleccionados manual o automáticamente a través del histograma de la imagen. En el segundo caso, primero se detectan bordes dentro de la imagen y luego en una serie de etapas, se generan curvas cerradas limitantes para completar los segmentos. En el caso de los métodos basados en regiones se utilizan procesos de crecimiento, división y mezclado, o sus combinaciones, para conectar espacialmente píxeles dentro de segmentos o regiones más o menos homogéneas. Las descripciones detalladas, asi como los antecedentes matemáticos y las evaluaciones de diversos algoritmos se encuentran recopilados por diferentes autores [4], [10], en la bibliografía consultada. En cualquier caso. una cuestión muy importante a tener en cuenta. es la evaluación del resultado de la segmentación y en este sentido, se han propuesto diferentes métricas [11].

Entre los métodos hibridos o mixtos que combinan la detección de bordes con el crecimiento de regiones, se encuentra el algoritmo propuesto por Adams y Bischof [12]. Este se inicia con la selección de un conjunto de píxeles denominados "semillas" a partir de las cuales las regiones van creciendo y mezclándose. Desde su propuesta, han aparecido investigaciones con el objetivo de optimizarlo [13]. [14].

En este trabajo se utiliza un método híbrido automático no supervisado (a diferencia de los basados en umbrales) de segmentación de imágenes multibanda. basado en crecimiento de regiones [15], y se propone como novedad frente a los existentes, una optimización en la selección evaluada de las semillas iniciales y de los parámetros del algoritmo de segmentación. Se proponen cuatro estrategias de selección y la función de evaluación objetiva propuesta [16] combina la varianza como indicador de homogeneidad global de las regiones y la autocorrelación espacial (índice I de Moran), como indicador de heterogeneidad entre las mismas. La propuesta se diferencia de los métodos OBIA estándar en que se evalúan cuatro estrategias de selección de las semillas iniciales.

La organización del trabajo es la siguiente: en la Sección II se explica la metodología de segmentación propuesta. La Sección III muestra el caso de estudio y un análisis de los resultados obtenidos. Finalmente, se pueden encontrar las conclusiones en la Sección IV. 


\section{Metodología De Segmentación}

\section{A. Crecimiento de regiones}

Esta metodología de segmentación consta de tres etapas que se especifican a continuación. La primera etapa es la generación automática de semillas de acuerdo a las diferentes estrategias que serán discutidas en la sección II.1. La segunda etapa es la de crecimiento de regiones, a partir de las semillas obtenidas de la etapa anterior. Por último, se aplica un algoritmo para fusionar las regiones que cumplen ciertos requisitos de similitud previamente especificados, con el fin de evitar el exceso de segmentación en la imagen. La Fig. 1 muestra el diagrama metodológico.

\section{1) Selección de semillas}

El algoritmo de crecimiento de regiones comienza por la elección de unos píxeles de partida o semillas. La forma más habitual es seleccionarlas eligiendo al azar un conjunto de píxeles de la imagen, o siguiendo una dirección de muestreo de la imagen, establecida a priori. Este algoritmo es muy sensible a las semillas iniciales, por lo que el resultado de la segmentación puede verse influido en gran medida por las semillas seleccionadas. Para obtener unos buenos resultados, estas semillas deben cumplir una serie de requisitos, como el hecho de que no deben situarse en zonas de ruido o en zonas de bordes. Y además hay que tener en cuenta algunas condiciones espectrales, y es que, las semillas deben ser representativas de los objetos significativos y homogéneos, finalmente obtenidos como resultado de la segmentación. Teniendo en cuenta esta idea, en esta investigación se plantean cuatro estrategias, que serán evaluadas posteriormente.

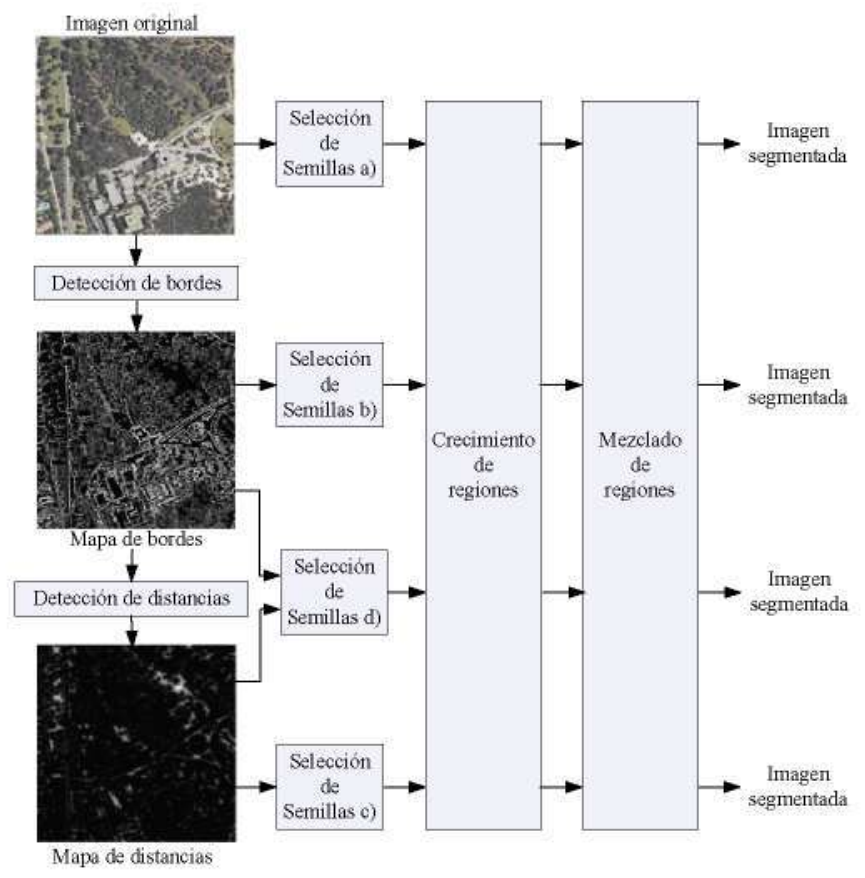

Figura 1. Diagrama metodológico del proceso de segmentación.

a) Selección aleatoria:

Las semillas se seleccionan de acuerdo con un muestreo de la imagen por filas, de manera aleatoria.

\section{b) Selección basada en bordes:}

Las semillas se seleccionan a partir de un mapa de bordes que se ha generado a partir de dos etapas de procesado de imagen. En primer lugar, se realiza un suavizado por convolución gaussiana con el fin de reducir el ruido $\mathrm{y}$ posteriormente, en una segunda etapa, se aplica el operador Sobel [17] para extraer bordes. Este operador se utiliza porque es eficaz, una vez reducido el ruido, debido a que presenta un bajo coste computacional, cuestión importante si se trabaja con escenas multibanda (RGB), con alta resolución espacial, calificadas en Teledetección, como escenas "pesadas" computacionalmente.

\section{c) Selección basada en distancias:}

Las semillas se seleccionan a partir de un mapa de distancias generado a partir del mapa de bordes comentado anteriormente. En una siguiente etapa, se realiza una umbralización con histéresis, que consiste en la imposición de dos umbrales, bajo y alto, con el objetivo de eliminar ruido pero sin romper los contornos. Estos umbrales se seleccionan automáticamente del histograma de la imagen inicial. Finalmente, se trasforma el mapa de bordes en mapa de distancias utilizando un algoritmo chamfer como se sugiere en [17], calculando y representando la distancia de cada píxel al borde más cercano. Finalmente, los píxeles con valor de distancia máxima al píxel del borde más cercano serán seleccionados como semillas.

\section{d) Selección basada en un método mixto usando bordes $y$ distancias:}

Las semillas se seleccionan a partir de un mapa generado por la combinación del mapa de bordes y del mapa de distancias. Esta combinación se puede realizar añadiendo al mapa de bordes, el de distancias. En caso de utilizar la estrategia del mapa de bordes, los píxeles con valor de gradiente mínimo eran considerados como las mejores semillas, mientras que en el caso del mapa de distancias, los píxeles con valor de la distancia máxima al píxel de borde más cercano, eran considerados como las mejores semillas. Por lo tanto, en esta estrategia, se propone que las distancias en su mapa tienen que ser modificadas de tal manera que valores positivos pasarán a valores negativos, para agregarlo al mapa de bordes. Así, los píxeles con valores mínimos serán seleccionados como semillas.

\section{2) Crecimiento de regiones}

Una vez que el conjunto de semillas se ha establecido, las regiones crecen por adición sucesiva de píxeles vecinos en las diferentes direcciones, que se consideran similares de acuerdo con un cierto criterio de similitud, aumentando paso a paso el tamaño de cada región. En esta investigación, el criterio adoptado es que la diferencia de valor digital entre el píxel y el promedio de la región es menor que un valor especificado por el usuario denominado umbral de similitud. Para evaluar esta diferencia se consideró calcular la Distancia Euclídea 
considerando todas las bandas en la imagen. Esta distancia puede ser considerada como medida de similitud [18] y entre todas las métricas de uso generalizado para el cálculo de la distancia entre valores digitales de pixeles de una imagen, es la de más bajo costo de cálculo [4], es resistente al ruido (ya que promedia las muestras a través de todas las bandas espectrales), y es reversible. La ecuación I. muestra la expresión matemática que se utiliza para el cálculo del valor de la distancia,

$$
d(x, y)=\sqrt{\sum_{i=1}^{n}\left(x_{t}-y_{i}\right)^{2}}
$$

donde $x_{t}$ es el valor espectral del píxel vecino en la banda i e $y_{t}$ es el valor espectral medio de la región de banda $\mathrm{i}$.

El proceso de crecimiento de regiones se continúa hasta que un píxel no es suficientemente similar como para ser agregado a una región dada. Esto significa que el píxel pertenece a otra región y el proceso de crecimiento en una dirección está acabado. Cuando no hay píxeles vecinos en cualquier dirección. que puedan ser incluidos en la región. el proceso se ha completado. Un inconveniente que se presenta con cierta frecuencia en los algoritmos de segmentación de imágenes es que tienden a generar un número significativo de las regiones pequeñas, que por lo general corresponden a píxeles de transición (como los bordes a lo largo de las fronteras entre las regiones), o a los objetos que son demasiado pequeños debidos a la resolución espacial de la imagen. Este problema se conoce como sobre-segmentación y para minimizarlo, se aplican algoritmos de mezclado de regiones.

\section{B. Mezclado de regiones}

El algoritmo de mezclado de regiones propuesto en este estudio incluye dos etapas básicas. En la primera etapa, todas las regiones con área (número de píxeles) menor o igual que 5 (este parámetro ha sido elegido después de varios experimentos previos) desaparecerán (individualmente) mediante su unión con su región vecina mas similar. Este paso es importante para evitar que se obtengan regiones muy pequeñas cuyo origen suele estar en el ruido presente en las imágenes. La Distancia Euclídea dada por la ecuación (1) se puede utilizar como medida de similitud, pero en este caso se calculará a partir de los valores medios de ambas regiones.

En la segunda etapa, todas las regiones con un área menor que el valor especificado por el usuario, que no se hayan fusionado en la etapa anterior, se unirán con las vecinas según un criterio de homogeneidad que será especificado en el siguiente apartado.

\section{Segmentación y evaluación conjunta}

Cabe señalar que, en cualquier método que se adopte para realizar la segmentación de una imagen. un paso fundamental es la selección de los parámetros del algoritmo. En este caso tienen que establecerse dos parámetros tal y como se comentó en el apartado $A$. En la mayoría de los casos, estos parámetros son seleccionados mediante una estrategia prueba y error, esperando a conocer la validez de dicha selección al final del proceso, cuando se determina la calidad de la imagen segmentada por cualquiera de los métodos establecidos.

Sin embargo, en este estudio se plantea realizar una selección de parámetros conjuntamente con su evaluación, enfoque que proporciona una solución alternativa a la opción de prueba/error de otros algoritmos de segmentación. Así, se propone utilizar una función de evaluación objetiva que permite ir evaluando la homogeneidad intra-segmento y la heterogeneidad inter-segmento, mientras se van realizando las diferentes etapas para la generación de regiones. En esta investigación, se utiliza una función de evaluación propuesta por Spindola [16], que combina un indicador como la varianza de homogeneidad global de las regiones y un indicador de autocorrelación espacial que detecta la separabilidad entre las mismas.

El primer componente de la función de evaluación es la varianza intra-segmento de las regiones y es calculada mediante la ecuación (2):

$v=\frac{\sum_{i=1}^{n}\left(a_{i} \times v_{i}\right)}{\sum_{i=1}^{n}\left(a_{i}\right)}$

donde $v_{i}$ es la varianza y $a_{i}$ es el área de la región i.

La varianza intra-segmento es una media ponderada, donde las ponderaciones son las áreas de cada región. Esta aproximación considera que las regiones más grandes, participarán con más peso, evitando así las posibles inestabilidades causadas por las regiones más pequeñas.

Para evaluar la heterogeneidad entre segmentos, la función utiliza el índice de correlación I de Moran, que mide el grado de asociación espacial reflejado en el conjunto de datos de manera global y es calculado mediante la expresión de la ecuación (3):

$$
I=\frac{n \sum_{i=1}^{n} \sum_{j=1}^{n} w_{i j}\left(y_{i}-\bar{y}\right)\left(y_{j}-\bar{y}\right)}{\left(\sum_{i=1}^{n}\left(y_{i}-\bar{y}\right)^{2}\right)\left(\sum_{i \neq j} \sum\left(w_{i j}\right)\right)}
$$

donde $n$ es el número total de regiones, $w_{i j}$ es una medida de la proximidad espacial, $v_{i}$ es el valor medio del nivel digital de la región $R_{i \star}$ e $y$ es el valor medio del nivel de gris (valor digital) de la imagen. Cada peso $w_{i j}$ es una medida de la adyacencia espacial de regiones $R_{i}$ y $R_{j}$. Así. si las regiones $R_{i}$ y $R_{j}$ son adyacentes, $w_{i j}=1$. De lo contrario, $w_{i j}=0$.

La elección adecuada de los parámetros de segmentación será la que condızca a un bajo índice inter-segmentos I de Moran (regiones adyacentes son muy diferentes) con una varianza intra-segmento baja (cada región es más homogénea). Para combinar ambos indicadores en la función objetiva de evaluación, la varianza y la medida de autocorrelación, hay que realizar una normalización previa para ambos componentes, entre 0 a 1 ; por lo tanto, los valores que tome esta función estarán finalmente en un rango entre 0 y 2. Cuanto más se aproxime al valor máximo. el resultado de la segmentación será considerado más óptimo. Esta función 
objetiva de evaluación es expresada por la ecuación (4):

$$
F(v, I)=F(v)+F(I) \quad ; \quad F(x)=\frac{X_{\max }-X}{X_{\max }-X_{\min }}
$$

donde $F(v)$ y $F(I)$ son funciones de normalización.

El método de segmentación-evaluación conjunta de la imagen, propuesto en este estudio, consiste en generar una secuencia de crecimiento-mezclado de regiones con un incremento del umbral de similitud de 10 por cada prueba, considerando valores de este umbral en el intervalo de 0 a 100 . Esta secuencia se repite para tres valores posibles del parámetro mínimo de población, definido como un porcentaje del número de píxeles de la imagen de entrada, específicamente los valores seleccionados para mostrar los resultados de este estudio son $0,0,2$ y $0,4 \%$. De esta manera, la función de evaluación descrita estima la calidad de cada segmentación, conjuntamente con la determinación de los valores de los parámetros óptimos.

\section{CASO DE ESTUDIO}

\section{A. Caracteristicas de la imagen}

Se trata de una imagen multibanda de tres bandas, con un tamaño de $1024 \times 1024$ píxeles, registrada mediante un sensor aéreo (resolución espacial $=0,5 \mathrm{~m}$ ). La imagen es de 8 bits, de tal manera que la respuesta espectral de los diferentes píxeles esta codificada entre 0 y 255 niveles de gris (niveles digitales).

En esta escena (Fig. 2), se observan mediante análisis visual experto, especies de vegetación mediterránea que coexisten con praderas naturales, suelo desnudo y un conjunto de clases de cubiertas terrestres generadas por efectos antropogénicos, como caminos asfaltados de algunas urbanizaciones con viviendas, zonas de recreo (piscina) y aparcamientos. Esto le confiere a la escena en su conjunto, bastante heterogeneidad espectral, debido a la coexistencia de diferentes coberturas de terreno.

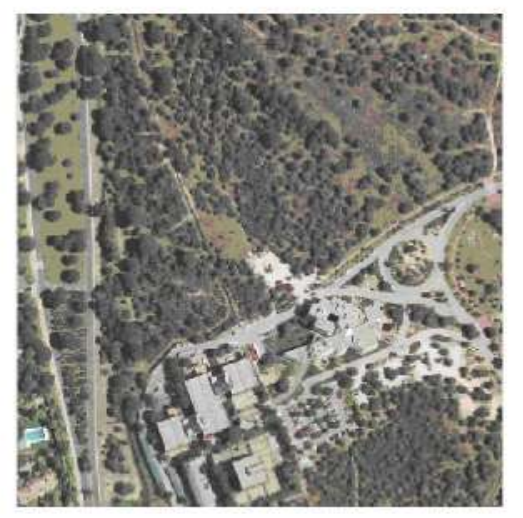

Figura 2. Composición color natural (RGB 321) de la escena de estudio.

\section{B. Análisis de Resultados}

Con el fin de evaluar las diferentes estrategias de selección de semillas, con un bajo coste computacional, se han seleccionado dos subescenas (Fig. 3) de $368 \times 368$ píxeles (aproximadamente $10 \%$ de píxeles de la imagen) de acuerdo con la mayor o menor heterogeneidad juzgada mediante análisis visual. La Escena 1 representa la mayor heterogeneidad y la 2 la menor heterogeneidad.

El procedimiento de análisis para ambas imágenes es el mismo y la metodología es la descrita en la sección II, tanto para la segmentación como para la evaluación de las regiones, prevista en las diferentes etapas. Desde un punto de vista computacional, los resultados se obtienen con un procesador a $2,1 \mathrm{GHz}$ y $640 \mathrm{MB}$ de RAM.

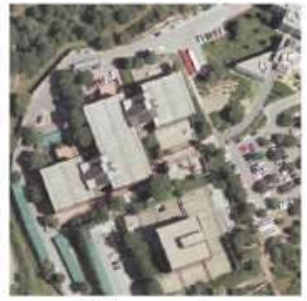

Escena 1

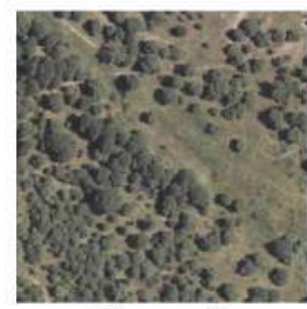

Escena 2
Figura 3. Composición color natural (RGB 321) de las dos subescenas (Escena 1 y Escena 2) seleccionadas.

\section{1) Escena I}

La representación tridimensional de la función objetiva $(\mathrm{Z})$ frente a los dos parámetros que evalúa (población mínima, $\mathrm{Y}$, y umbral de similitud, X) es mostrada (Fig. 4) conjuntamente con el resultado de la imagen segmentada mostrando los parámetros óptimos (según el valor máximo de la función objetiva). Hay que indicar que los segmentos obtenidos se han coloreado según su nivel digital medio, de acuerdo con la composición RGB correspondiente a la imagen de origen.

Observando dicha figura, cabe señalar que, existe una dependencia significativa de la calidad de la segmentación, con el parámetro de similitud y el de población mínima. Según se muestra en las graficas de la Fig. 4, independientemente del método de selección de semillas adoptado para la segmentación, el valor máximo de la función de evaluación se obtiene para un umbral de similitud de 40 y una mínima población del $0,2 \%$. Por otro lado hay que indicar que la selección de semillas usando el mapa de bordes y la estrategia mixta genera el mayor valor para la función de evaluación, 1,388 y 1,384 , respectivamente, tal y como se muestra en las Fig, $4 \mathrm{~b}$ y $4 \mathrm{~d}$.

Estos valores máximos coinciden con la interpretación visual de las imágenes segmentadas de la Fig. 4. Se muestra que se ha logrado un equilibrio entre la sub y la sobresegmentación, tal y como se resalta en el círculo rojo de las imágenes segmentadas resultantes, tanto en el caso de la estrategia basada en selección de semillas en mapa de bordes (Fig. 4b) como en la estrategia mixta (Fig. 4d). Estas valoraciones subjetivas procedentes de una visualización de imágenes resultantes han sido supervisadas en la imagen original (Fig. 3).

Si se tiene en cuenta la segmentación óptima (con la mejor función objetivo), una sola ejecución del algoritmo presenta un tiempo de cálculo de 1,921 segundos y un uso de memoria de 74 MB. Por otro lado, la segmentación automática teniendo en cuenta todas las iteraciones presenta un tiempo de cálculo de 1,14 minutos y el mismo uso de la memoria. 


\section{2) Escena 2}

Los resultados obtenidos para el caso de la función de evaluación para cada estrategia de selección de las semillas se exponen en la Fig. 5. En dicha figura, se vuelve a poner de manifiesto la dependencia significativa de la calidad de la segmentación, con los parámetros utilizados en el proceso, mostrando en la gráfica en este caso, como resultado, dos máximos en el valor de la función de evaluación.

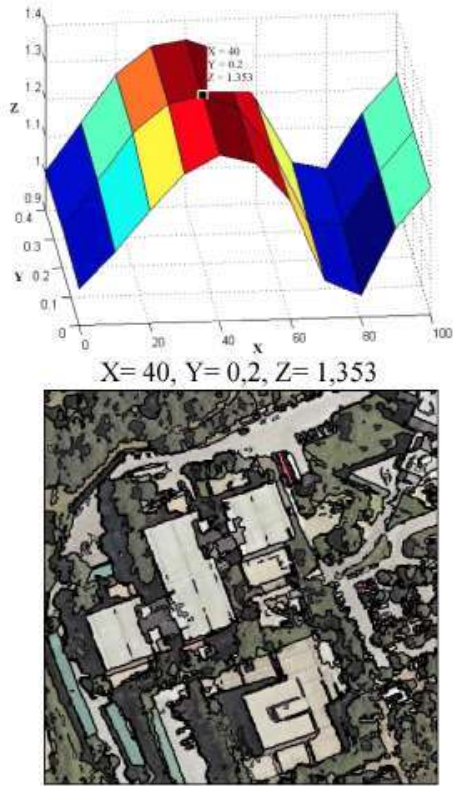

(a)

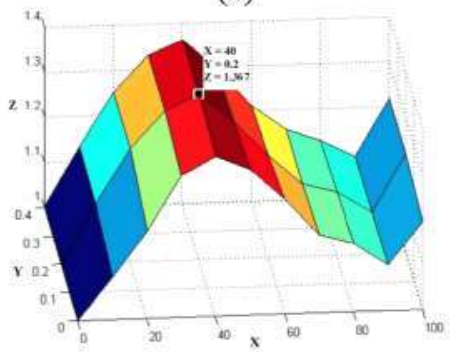

$\mathrm{X}=40, \mathrm{Y}=0,2, \mathrm{Z}=1,367$

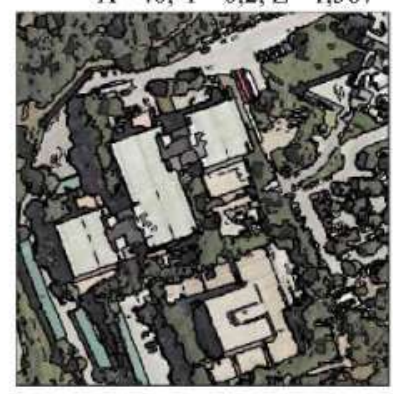

(c)
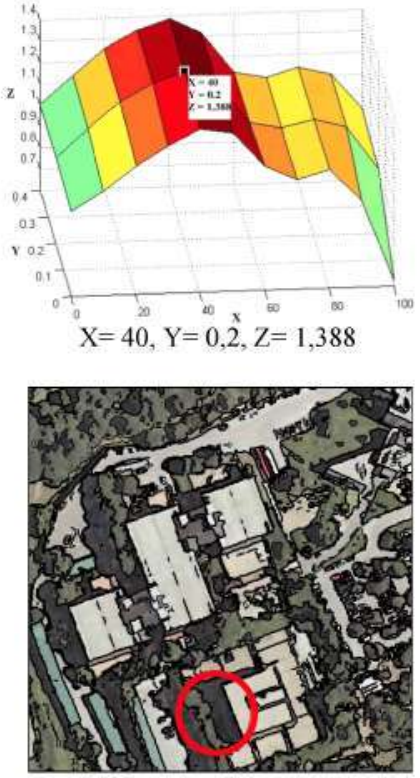

(b)
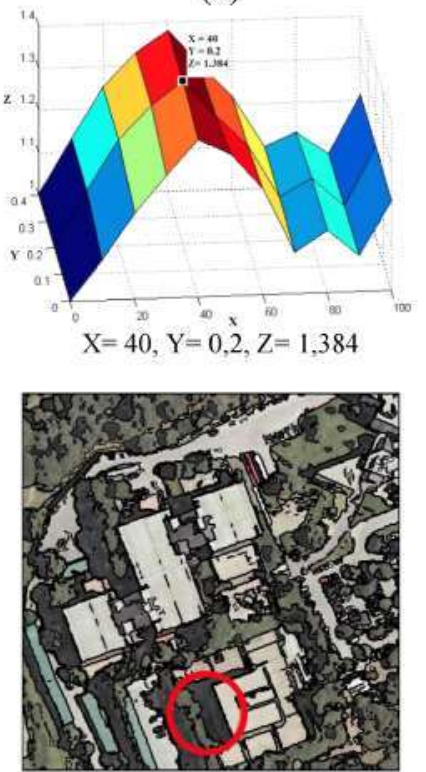

(d)
Figura 4. Resultados sobre la Escena 1. Representación tridimensional de la función objetiva de evaluación frente a los parámetros de población mínima y umbral de similitud, junto con la imagen segmentada para cada estrategia de selección de semillas. (a) Selección aleatoria, (b) Selección basada en mapa de bordes, (c) Selección basada en el mapa de distancias y (d) Selección basada en una estrategia mixta.

El valor más alto de dicha función aparece en el caso de un umbral de similitud de 50 y un mínimo de población de un $0,2 \%$, con valores de la función objetiva superiores a los obtenidos en la Escena 1, tanto para la estrategia de selección de semillas en el mapa de bordes (Fig. 5b), como en la estrategia mixta (Fig. 5d). Los resultados de las imágenes segmentadas con estos parámetros ofrecen una subsegmentación de algunas regiones. En la Fig. 6 se muestra la Escena 2 segmentada mediante la estrategia de selección de semillas mediante bordes, para los valores óptimos de similitud de 30 y 50 . Se observa que para el caso del valor del umbral igual a 50 hay una sub-segmentación de la imagen y para el caso del valor igual a 30, hay sobre-segmentación.

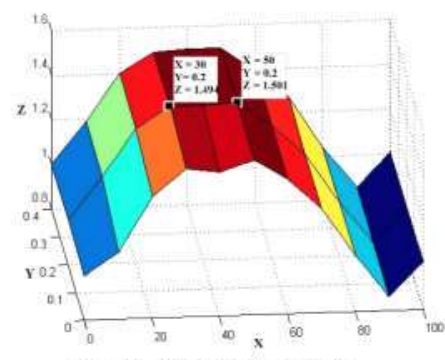

$\mathrm{X}=30, \mathrm{Y}=0,2, \mathrm{Z}=1,494$

$\mathrm{X}=50, \mathrm{Y}=0,2, \mathrm{Z}=1,501$

(a)

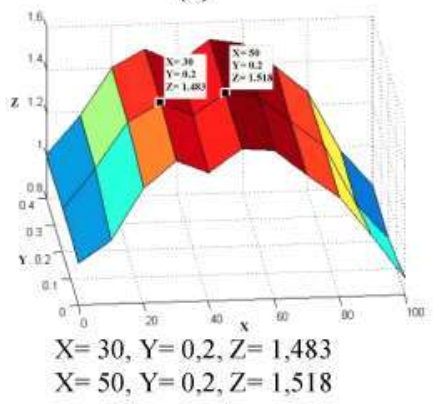

(c)

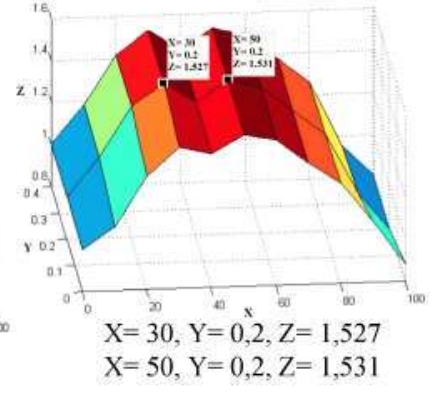

(b)

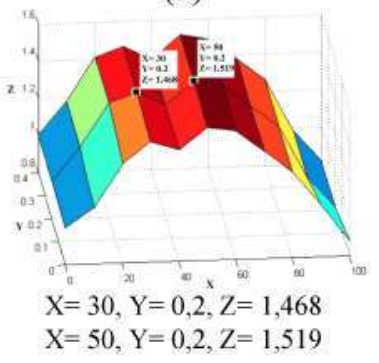

(d)
Figura 5. Resultados sobre la Escena 2. Representación tridimensional de la función objetiva de evaluación frente a los parámetros de población mínima y umbral de similitud para cada estrategia de selección de semillas. (a) Selección aleatoria, (b) Selección basada en mapa de bordes, (c) Selección basada en el mapa de distancias y (d) Selección basada en una estrategia mixta.

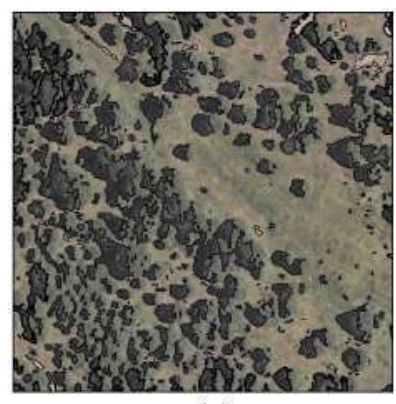

(a)

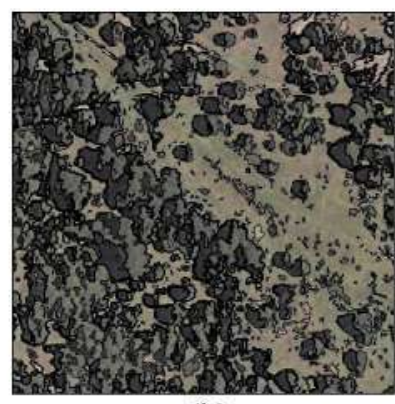

(b)
Figura 6. Representación de la imagen segmentada de la Escena 2 para la estrategia de selección de semillas mediante bordes, con parámetros óptimos de umbral de similitud y $0,2 \%$ de población. (a) umbral de similitud igual a $50 \mathrm{y} \mathrm{b}$ ) umbral de similitud igual a 30 .

Una posible causa a considerar, es que esta escena es bastante homogénea y hay poca diferencia espectral entre las regiones, por lo que el índice I de Moran aumentará ligeramente de una iteración a otra. En adelante se considera como válido un valor 
del umbral de similitud de 40 , manteniendo el valor del mínimo de población al $0,2 \%$, ya que la función de evaluación no disminuye ostensiblemente, según se muestra en la Fig. 5b y $5 \mathrm{~d}$.

El estudio completo se repite para la imagen completa (Fig. 2), para comprobar la idoneidad de la selección de las subescenas y de manera concluyente, se comprueba que los parámetros óptimos son, 40 como valor umbral de la similitud y $0,2 \%$ como población mínima. La estrategia óptima para la generación del conjunto de semillas iniciales resulta ser la que utiliza el mapa de bordes. El resultado de dicha segmentación es el mostrado en la Fig. 7.

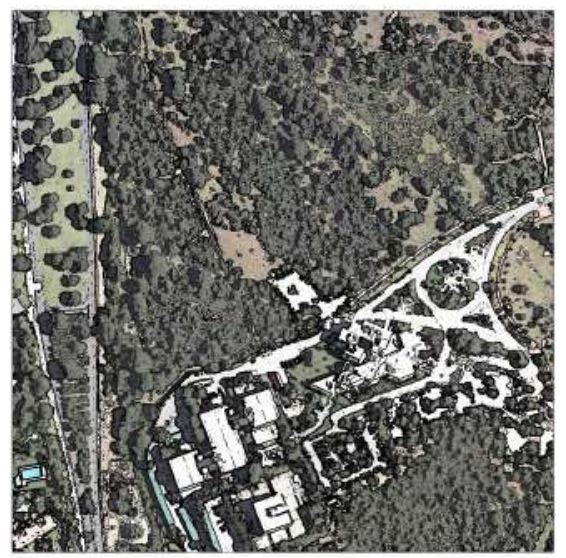

Figura 7. Imagen segmentada con los parámetros de población mínima igual a $0,2 \%$ de población de la escena y umbral de similitud igual a 40 , para la estrategia de selección de semillas a partir del mapa de bordes.

Una inspección visual exhaustiva de la imagen segmentada, indica que los segmentos obtenidos corresponden a su distribución espacial en la imagen original. La función de evaluación objetiva tiene un valor de 1,4 . Por lo tanto, los parámetros seleccionados nos han asegurado una buena calidad de la segmentación con regiones u objetos espectral y espacialmente homogéneos. El tiempo de proceso total resultó ser de 9 segundos y se utilizaron 95 MB de memoria RAM.

\section{3) Aplicación de estrategia a Imagen QuickBird (QB)}

Un nueva imagen de dimensiones de $412 \times 336$ píxeles, registrada por QuickBird (resolución espacial: $2,44 \mathrm{~m}$ en nadir $\mathrm{y}$ resolución espectral: 4 bandas), fue seleccionada para realizar el estudio (Fig. 8a). Dicha imagen, originalmente codificada en 11 bits, se preprocesó para trabajar con codificación en 8 bits.

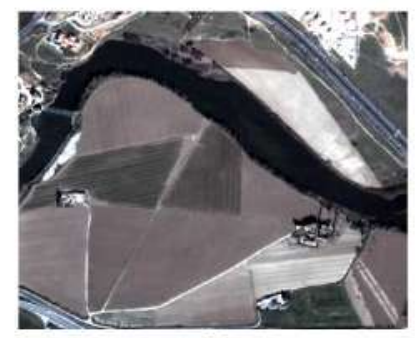

(a)

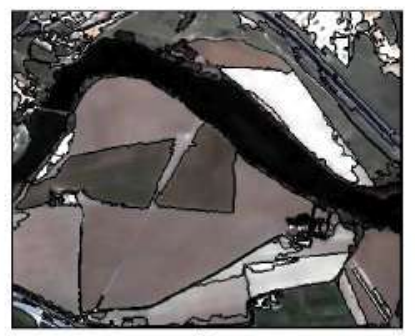

(b)
Figura 8. (a) Imagen QB original y (b) Imagen QB segmentada con los parámetros de población mínima igual a 138 y umbral de similitud igual a 10 , para la estrategia de selección de semillas a partir del mapa de bordes.
Una vez realizado el estudio de evaluación, a la nueva imagen se le aplicó la misma estrategia de crecimiento de regiones a partir de semillas obtenidas desde el mapa de bordes. De acuerdo con la función de evaluación óptima, los valores seleccionados fueron: 10 para el umbral de similitud, y 138 pixeles para el valor mínimo de población. El resultado de la segmentación de QB está representado en la Fig. 8b.

Una inspección visual exhaustiva de la imagen segmentada (Fig. 8b) indica que se han obtenido buenos resultados. El coste computacional en una sola iteración del algoritmo ha sido prácticamente el mismo que en los casos anteriores de las Escenas 1 y 2 (2 s, 74MB).

\section{4) Comparación de resultados con la segmentación multi- resolución.}

Las imágenes aérea y QB son segmentadas mediante el algoritmo de segmentación multi-resolución [19], muy común en OBIA, implementado en el software eCognition (B). En este algoritmo los píxeles se adhieren unos a otros a partir de unas semillas iniciales siguiendo un criterio de homogeneidad, basado en tres parámetros ajustables: factor de escala (parámetro de control del tamaño de los objetos), shape/color (peso de la información espectral en la segmentación) y compactness/smoothness (peso de la información morfológica de los objetos, suavidad de contornos y compacidad en la formación de los objetos).

El resultado óptimo para la imagen aérea, representado en la Fig. 9, se obtuvo con los valores de parámetros de segmentación siguientes: 100 para factor de escala, 0,1/0,9 para forma/color y $0,7 / 0,3$ para la información morfológica.

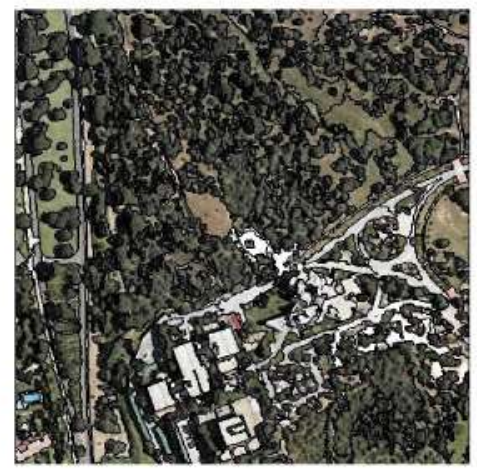

Figura 9. Imagen segmentada mediante el algoritmo de segmentación multiresolución de eCognition. Los parametros de homogeneidad son: 100 para factor de escala, 0,9 es el peso para color y 0,7 para la forma.

Para el caso de la imagen QB, el resultado óptimo se representa en la Fig. 10 y se obtuvo con los valores de parámetros de segmentación siguientes: 100 para factor de escala, $0,1 / 0,9$ para forma/color y 0,3 para la información morfológica.

Mediante análisis visual exhaustivo de las imágenes de las Fig. 9 y 10, se observa que presentan algunas pequeñas diferencias con zonas de sobre-segmentación, con respecto a los resultados de las Fig. 7 y 8 . El tiempo de cómputo ha sido de 24 segundos para la aérea y 10 segundos para la QB.

Los valores mayores para el tiempo de proceso en este caso, se justifican por el mayor número de variables que 
intervienen en el proceso de segmentación multi-resolución.

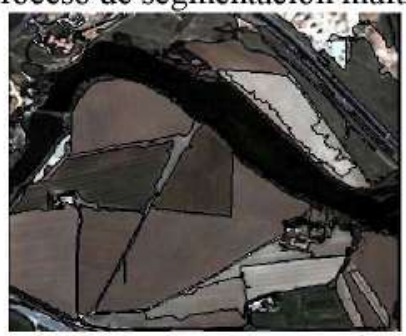

Figura 10. Imagen segmentada mediante el algoritmo de segmentación multiresolución de eCognition. Los parámetros de homogeneidad son: 100 para factor de escala, 0,9 es el peso para color y 0,7 para la forma.

\section{CONClusiones.}

En este trabajo se ha presentado una metodología objetiva de selección y evaluación conjunta, de los parámetros de segmentación de un algoritmo no supervisado, de crecimiento de regiones. Se han presentado y evaluado cuatro estrategias de selección de semillas iniciales, paso que se considera crítico en este tipo de segmentación, lo que se ha confirmado a lo largo del estudio. Los resultados experimentales sobre diferentes imágenes multibanda, con diferente variabilidad espectral reflejada en los niveles digitales han resultado buenos en los cuatro casos; sin embargo, la estrategia del mapa de bordes ha sido la mejor. La estrategia propuesta es adecuada para imágenes de diferente resolución espectral y espacial, como se ha podido comprobar con los resultados de la imagen QuickBird.

Hay que hacer notar que el coste computacional ha sido bajo, comparado con el utilizado por el algoritmo multiresolución de eCognition. Este hecho puede ser debido a la diferente estrategia de segmentación de ambos algoritmos, y al hecho de que se ha diseñado el software que contiene nuestro algoritmo, optimizando al máximo su programación.

Sin embargo, para imágenes de mayores dimensiones, se propone paralelizar el proceso, debido a que la segmentación propuesta es inherentemente paralelizable. Esto constituirá la futura línea de investigación, derivada de este estudio.

\section{REFERENCIAS}

[1]T. Blashke, "Object Based Image Analysis For Remote Sensing", ISPRS Journal of Photogrammetry and Remote Sensing, vol. 65, no. 1, pp. 2-16, 2010.

[2]S. W. Myint, P. Gober, A. Brazel, S. Grossman-Clarke and Q. Weng, "Perpixel vs. object-based classification of urban land cover extraction using high spatial resolution imagery", Remote Sensing of Environment, 115, pp. 11451161, 2011.

[3]U. C. Benz, P. Hofmann, G. Willhauck, I. Lingenfelder and M. Heynen, "Multi-resolution, object-oriented fuzzy analysis of remote sensing data for GIS-ready information", ISPRS Journal of Photogrammetry and Remote Sensing, vol. 58, no. 3-4, pp. 239-258, 2004.

[4]N. R. Pal, and S. K. Pal, "A Review on Image Segmentation Techniques", Pattern Recognition, vol. 26, no. 9, pp. 1277-1294, 1993.

[5]C. A. Glasbey and G. W. Horgan, Image analysis for the biological science, Statistics in Practice, Series Editor Vic Barnett., Jonh Wiley and Sons, 1994.

[6]J. S. Suri, D. Wilson and S. Laxminarayan, Handbook of Biomedical Image Analysis. Vol. 1: Segmentation Models Part A, Topics in Biomedical Engineering. International Book Series, E. Micheli-Tzanakou (ed.), 2005.

[7] M. A. Gonzalez and V. L. Ballarin, "Segmentación de imágenes utilizando la transformada Watershed: obtención de marcadores mediante lógica difusa", IEEE Latin America Transactions, vol. 6, no. 2, pp. 223-228, 2008.
[8]M. A. Gonzalez, G. J. Meschino and V. L. Ballarin, "Solving the over segmentation problem in applications of Watershed Transform", Journal of Biomedical Graphics and Computing, vol. 3, no. 3, pp. 29-39, 2013.

[9]Z. Ma, J.M.R.S. Tavares, R. Natal, J. and T. Mascarenhas, "A review of algorithms for medical image segmentation and their applications to the female pelvic cavity", Computer Methods in Biomechanics and Biomedical Engineering, vol. 13, no. 2, pp. 235-246, 2010.

[10] R. C. Gonzalez, and R. E. Woods, Digital Image Processing, 2nd Edition, Ed. Prentice Hall, 2002.

[11] M. Neubert, H. Herold, and G. Meinel, "Assessing Image Segmentation Quality - Concepts, Methods and Applications" in: Object-Based Image Analysis - Spatial concepts for knowledge-driven remote sensing applications. T. Blaschke, G. Hay, S. Lang, (Eds.). Springer, Berlin, 2008.

[12] R. Adams and L. Bischof, "Seeded region growing", IEEE Transactions on Pattern Analysis and Machine Intelligence, vol. 16, no. 6, pp. 641-647, 1994.

[13] J. Fan, G. Zeng, M. Body and M. Hacid, "Seeded region growing: an extensive and comparative study", Pattern Recognition Letters, vol. 26, no. 8, pp. 1139-1156, 2005.

[14] J. Liu, Y. Ma, K. Chen and S. Li, "A Novel Color Image Segmentation Method Based on Improved Region Growing", Lecture Notes in Computer Science, Artificial Intelligence and Computational Intelligence, vol. 7530, pp. 365-373, 2012.

[15] J. Sanchez, E. Martinez, A. Arquero, and D. Renza, "Automatic Image Segmentation Optimized by Bilateral Filtering". CIARP'10, Lecture Notes in Computer Science, vol. 6419, 303-310, 2010.

[16] G.M. Spindola, G. Camara, I. A. Reis, L.S. Bins, and A. M. Monteiro, "Parameter selection for region-growing image segmentation algorithms using spatial autocorrelation", International Journal of Remote Sensing, vol. 27, no. 14, pp. 3035-3040, 2006.

[17] W. Burger and M., Burge. Digital Image Processing: An Algorithmic Introduction Using Java. Texts in Computer Science, Ed. Springer, 2012.

[18] S. Santini, and R. Jain, R, "Similarity measures", IEEE Trans. on Pattern Analysis and Machine Intelligence, vol. 21, no. 9, pp. 871-883, 1999.

[19] M. Baatz and A. Schäpe, "Multi-resolution segmentation - an optimization approach for high quality multi-scale segmentation", in: Strobl, J et al. (Hrsg.): Angewandte Geographische Informations verarbeitung XII, 1223. Beiträge zum AGIT Symposium Salsburg 2000, Karlruhe, Herbert Wichmann Verlag. 2000. 